\title{
ATLAS LINGUISTIQUE DE LA PARAÍBA ${ }^{1}$
}

\author{
VERSÃO FRANCESA DO ORIGINAL \\ ATLAS LINGUÍSTICO DA PARAÍBA
}

Maria do Socorro Silva de Aragão

Le projet inicial de l'Atlas remonte à 1974 mais c'est deux ans plus tard que devait débuter la formation technique des chercheurs devant prendre part aux enquêtes : certains d'entre eux, bénéficièrent de séjours d'études, en France et en Espagne, pays ayant une longue tradition dans les recherches dialectales, pour approfondir leur connaissances sur les approches théoriques de la dialectologie et de la géographie linguistique, et entrprendre des recherches bibliographiques. Les travaux de l'Atlas Linguistique de Paraíba (ALPB) ont débuté concrètement, de manière efficace et systématique, en janvier 1979: l'œuvre devait comporter trois volumes, dont deux ont déjà été publiés.

Le premier volume, l'Atlas proprement dit, comporte 149 cartes, lexicales et phonétiques, précédées de la présentation générale de l'ouvrage, de l'approche méthodologique utilisée et de la transcription phonétique (voir ci-après).

Le deuxième volume compred, avec un court historique du projet, la liste les localités du réseau et des informateurs et, sur le plan linguistique, une analyse des structures phonético-phonologiques (voir ci-après) et morpho syntaxiques du parler Paraíbano. Il comporte aussi un glossaire rassemblant, en particulier, les formes employées le plus fréquemment dans l'Etat de Paraiba même si elles peuvent aussi être attestées dans d'autres régions; celles qui figurent dans les dictionnaires officiels mais avec un autre sens, ainsi que les termes qui ne figurent pas dans ces derniers. On y trouve enfin une liste de références bibliographiques.

Un troisième volume, avec les données collectées à partir d'un questionnaire spécifique, est en attente de publication.

1 In: Geolinguistique Paris: Université de Paris Sorbonne , v.101, p.37 - 53, 2015

Vol. 25 - Ano $44-n^{\circ} 1-2020$ 


\section{Présentation de l'Atlas}

\section{Le réseau}

Le réseau comprend 25 communes de base ${ }^{2}$, chacune comportant trois 'municipalités -satélites', sur un total de 100 communes, couvrant l'ensemble de l'État. Les 'municipalités- satélites' ont été utilisées pour le contrôle et la validation des données relevées dans les municipalités de base, sans que les résultats soient cartographiés.

Une enquête a été réalisée auprès de la Fondation de l'Institut Brésilien de Géographie et Statistiques - IBGE, permettant de préciser: a. les données géographiques et socio-économique-culturelles de toutes les localités retenues pour le réseau ${ }^{3} ; b$. les aspects démographiques ${ }^{4}$; les aspects économiques ${ }^{5}$; les aspects socioculturels ${ }^{6}$ : les moyens de transport et les routes d'accès; les échanges commerciaux et culturels, et d'autres informations.

\section{Le questionnaire}

La version du questionnaire utilisé, publié en 1980 par l'Éditeur de l'UFPB, est le résultat de l'amélioration de trois autres versions testées dans six communes, avec 12 locuteurs différents. Il a été divisé en deux parties : une partie générale, avec 289 questions, et une partie spécialisée, contenant 588 questions. Pour la sélection des référents des cartes ont été retenus plusieurs critères et, en particulier, la représentativité du parler régional, la richesse de la variation lexicale et phonétique et la possibilité de comparaison avec d'autres Atlas Linguistiques du Brésil. Ces référents sont devenus les sujets de chacune des cartes lexicales et phonétiques de l'Atlas. Le questionnaire général, prend en compte les champs sémantiques suivants : la terre, l'homme, la famille, le logement et le ménage, les oiseaux et les animaux, la culture de la terre pour la plantation et les activités sociales. Le questionnaire spécialisé, élaborées par les enseignants Alzir Oliveira, Otília Christophe Maia de Vasconcelos et Yara Lyra de Macedo de l'UFPB, concerne les désignations relatives aux cinq principaux produits agricoles de Paraíba: le manioc, la canne à sucre, le coton, l'agave et l'ananas.

\section{Les informateurs}

Le choix des 107 informateurs ayant participé aux enquêtes a obéi à plusieurs critères. Pour celles qui ont été réalisées avec la partie générale du questionnaire, ont été retenus des hommes ou des femmes nés dans le quartier ou dans la commune de l'enquête, d'âge compris entre 30 et 75 ans, mariés ou célibataires, analphabètes ou avec un niveau d'école primaire complet, ayant des parents nés dans la même région, ne pas avoir été absents de la localité pour plus d'un an, ayant une bonne élocution. Pour 
répondre au questionnaire spécifique les informateurs devaient exercer une une activité dans le domaine concerné par le champ sémantique choisi.

Dans chacune des communes, ont été interrogées trois personnes au minimum et dix au maximum, pour disposer d'un échantillon représentatif du parler local.

\section{Les enquêtes}

Les enquêtes ont été réalisées in loco, précédées d'un entretien préliminaire dans le but de mettre à l'aise les locuteurs avant de répondre aux questions. Pour faciliter la compréhension, les questions ont été formulées de façon simple, parfois avec la présentation dessins, d'objets ou encore avec l'imitation de certaines actions. Les enquêtes se sont déroulées soit chez les informateurs, soit sur leurs lieux de travail ou dans d'autres endroits comme l'Hôtel de Ville, le Centre Social Urbain, des locaux de coopératives ou d'associations. Les enquêtes ont toutes été enregistrées.

\section{La transcription phonétique}

Pour la transcription phonétique des enquêtes a été utilisé l'Alphabet Phonétique International, ayant un nombre réduit de signes et de signes diacritiques pour faciliter la lecture de l'ouvrage. La transcription phonétique est réalisée à partir de la seule impression auditive: il n'y a pas eu de contrôle instrumentale.

\section{Les cartes}

L'Atlas Linguistique de Paraíba commence par la carte de l'Etat de Paraíba et sa localisation dans le Brésil, sont présentées aussi les micro-régions homogènes, les divisions communales, les localités, les ethniques, les surnoms des habitants, les enquêteurs et les informateurs. Suivent les cartes linguistiques. Ces dernières ont été élaborées à l'aide de 68 questions relatives à des référents ayant montré le nombre d'occurrences le plus élevé et le plus grand nombre de variantes phonétiques et lexicales, regroupées par champs sémantiques (voir ci-après), l'ordre des cartes suivant celui des entrées du questionnaire.

- la terre :

- l'homme :

- la famille :

- la maison et le ménage :

- les oiseaux et les animaux :

- les cultures :

- les activités sociales : cartes $001-043$

cartes $044-090$

cartes 091-107

cartes $108-113$

cartes 114-129

cartes $130-137$

cartes 138-149.

Les cartes de l'ALPB, contrairement à la plupart des Atlas de première génération, sont des cartes à symboles : elles reflètent cependant la structure traditionnelle de ces 
derniers à la diférence près que, à côté de chaque point du réseau, on ne trouve pas les différentes désignations en trascription phonétique, mais les symboles correspondant à chacune d'entre elles présentées dans la légende, en orthographe standard. Dans un même point d'enquête on peut avoir, pour un référent donné, jusqu'à 8 symboles placés côte à côte, correspondant à autant de désignations différentes, et non pas un seul symbole correspondant une réponse unique, cas le plus fréquent dans la plupart des atlas (voir carte 083 'sovina', P. 22). En fait l'ordre des symboles ne serait pas tout à fait arbitraire dans la mesure où à chaque informateur connaissant plusieurs termes on a demandé de dire, en premier lieu, celui qui lui paraissait le plus caractéristique de sa région ou de l'Etat et de compléter ensuite sa réponse en énumérant les autres. L'ALPB montre laisse apparaître, pour certains champs sémantiques, une grande variété lexicale, pouvant aller jusqu'à 23 désignations différentes pour pour un référent donné (cartes 011 'vento suave' et 019 'garoa'). En même temps, cette situation laisse apparaître des difficultés à définir les véritables aires d'extension de chacune des formes relevées réparties dans tout le domaine d'enquête de façon irrégulière. Les exemples suivants témoignent de la richesse lexicale relative à quatre référents auxquels sont consacrées les cartes correspondantes et la structuration des désignation avec des formes simples (Mercùriu 'étoile filante'), composées (Sete estrelas 'id.') ou complexes (Deus te abrande 'étoile filante') que l'on peut voir dans la carte ci-après (038 'Estrela cadente') :

Arc-en-ciel: Ce référent répondant à la définition «Barres colorées qui apparaissent dans le ciel, avant ou après la pluie » - connaît, à Paraíba, 9 désignations : Arco-íris, Arco-celeste, Olho-de-boi, As barras, Sub-dourada, As torres, Os véus, Os vieiras, Arco. En tant que variantes phonétiques nous avons : ['afku 'iris]; ['awku 'iri] ; ['ajku 'iris] ; ['aku 'iris]; ['afku se'lefti]; ['ajku se'lefti] ; ['awku se'lefti] ; ['oKu di 'boy] ; ['oj di 'boy]; ['oyu di boy].

Etoile filante : les réponses à la question « Dans la nuit, souvent on peut observer une étoile qui traverse le ciel et laisse une trainée de lumière. Comment s'appelle ce phénomène?» ont montré l'existence de 14 designations (Estrela cadente, Estrela d'alva, Planeta, Zelação, Sete estrelas, Papa-ceia, Viração, Mercúrio, Barca, Rabisca, Elevação, Estrela mariana, Deus te abrande, Estrela se mudando) et de quelques variantes phonétiques: [if'trela ka' dêti] ; [if'trela 'dawva] ; [if'trela 'dalva] ; [if'trela 'davis].

Avare : La question concernant ce référent était : "Personne qui n'aime pas dépenser son argent et qui, parfois, connaît des difficultés pour ne pas le dépenser ». Les réponses ont montré l'existence de 21 désignations différentes dans l'espace enquêté (Amarrado, Unha-de-fome ; Pica-fumo ; Mesquinho, Sovina, Econômico, Chula, Fona, Somítico, Seguro, Fominha, Arrochado, Morto-a-fome, Usurário, Tacanha, Morto-defome, Papagaio-no-arame, Agarrado, Enforcado, Miserável, Resina ) ainsi que des variantes phonétiques de quelques unes d'entre elles: [su'vîna]; [suf'vînu] ; [kô'nômiku] [عkô'nômi] ; [mis'kînu] ; [mis'kîyu].

Vol. 25 - Ano $44-n^{\circ} 1-2020$ 
Cachasseiro: ce référent est represente par 13 désignations (Bêbado, Cachaceiro. Bebão, Embriagado, Encachaçado, Viciado, Pinguço, Melado, Alto do chão, Caneiro, Bebarrão, Puxando fogo, Alambique) et trois variantes phonétiques: ['bebadu] / ['bebedu]; ['bebu].

De nombreuses cartes lexicales sont complétées par des cartes qui relèvent les variantes phonétiques de certaines désignations, présentées en transcription phonétique dans la légende. Ainsi, à titre d'exemple, la carte 015 montre les 16 désignations du référent 'nublado' (question 21) et, la carte suivante, les 9 variantes phonétiques relevées pour le même mot.

\section{Analyse phonétique et phonologique}

Les données collectées au cours de enquêtes et présentées dans les cartes de l'atlas ont fourni la matière à une analyse phonético-phonologique - l'approche méthodologique adoptée est celle de l'Ecole de Prague - et morphosyntaxique des parlers de Paraiba, présentée, comme déjà signalé, dans le volume 2 intitulé "Analyse des formes et des structures linguistiques relevées». Les résultats, que nous présentons ci-après, permettent de comparer le phonétisme du portugais de cette région et celui du portugais standard.

\section{Tableaux des phonèmes}

Les résultats des analyses phonético-phonologiques des données recueillies sont résumés par les tableaux suivants présentant les phonèmes vocaliques, consonantiques et semivocaliques, les archiphonèmes ainsi que les les variantes libres et combinatoires.

\section{Phonèmes consonantiques et vocaliques oraux}

Tableau 01

\begin{tabular}{|c|c|}
\hline /p / / pata / «pata» & /b / / 'bata / «bata» \\
\hline /t / /'tcla / «tela» & / d / / 'dela / «dela» \\
\hline / k / / 'kala / «cala» & /g / / gala / «gala» \\
\hline /f / / 'fila / & / $\mathbf{v} /$ / 'vila / \\
\hline /s / / 'scla / & /z / /'zela / «zela» \\
\hline / $/$ / 'JatU / «chato» & / z/ / 'zatU / «jato» \\
\hline /1/ / 'mala / «mala» & / $\boldsymbol{\Lambda} /$ / 'maKa / «malha» \\
\hline$/ \mathbf{r} / \quad /$ murU / «muro» & / r / / 'murU / «murro» \\
\hline / a / / 'ala / «ala» & / $\boldsymbol{\varepsilon} /$ / 'ela / \\
\hline$/$ e / / ' elI / & / 'ira / \\
\hline$/ \mathbf{0} / /$ ' oKU / & /o / / 'ока / \\
\hline / u / / 'uva / & \\
\hline
\end{tabular}

\section{Phonèmes consonantiques et vocaliques nasals}

Tableau 02 


\begin{tabular}{|c|c|}
\hline / $\mathrm{m} /$ / 'mama / «mama» & / $\mathbf{n} / \quad$ / mana / «manha $\rangle$ \\
\hline / $\mathrm{n} /$ / 'mana / «mana» & / ̃̃ / /'mẽta / «menta» \\
\hline / ã / / 'mãta / & / $\tilde{\mathbf{o}} /$ / 'kõta / «conta» \\
\hline / $\tilde{1} /$ / 'sĩta / & / $\tilde{\mathbf{u}} /$ / 'zũta / «junta» \\
\hline
\end{tabular}

\section{Phonèmes semivocaliques}

Tableau 03

\begin{tabular}{|l|l|}
\hline$/ \mathbf{j} /$ / paj / «pai» & / w / / paw / «pau»
\end{tabular}

\section{Distribution des phonèmes}

Tous les phonèmes de la langue portugaise figurent aussi dans les parlers des informateurs de Paraíba. Dans leur réalisation sont présents cependant certains traits témoignant de la façon de parler Paraíbana : ainsi, dans certaines positions certaines oppositions sont neutralisées.

\section{Position initiale}

En position initiale sont admiss tous les phonèmes consonantiques à l'exception de /r / et / r/, dont l'opposition est neutralisée, et de / $\mathrm{n} /$ :

Tableau 4

\begin{tabular}{|c|c|c|c|c|}
\hline $\mid \mathbf{p} /$ & / paKa / «palha» & $7 \mathbf{b}$ & / bota $/$ & «bota» \\
\hline $7 \mathbf{t} /$ & /'tira / «tira» & $/ \mathbf{d} /$ & T'dedU & «dedo» \\
\hline$/ \mathbf{k} /$ & / kilu / & $\lg /$ & / gata / & «gata» \\
\hline $7 \mathbf{f} /$ & / faka / & $\mid \mathrm{v} /$ & T'vala / & «vala» \\
\hline $\mid \mathbf{s} /$ & / sala / & $|\mathbf{z}|$ & /'zelU / & «zelo» \\
\hline $7 \int 1$ & T'Juva / & 131 & T'zelU / & «gelo» \\
\hline Пॉ & ITlata / & $\mid\langle\overline{\mathbf{n}}$ & Ke / & 《he» \\
\hline$/ \mathbf{m} /$ & mata & $/ \mathbf{n} /$ & neta / & «neta» \\
\hline
\end{tabular}

\section{Position intervocalique}

Tous les phonèmes consonantiques peuvent figurer dans cette position:

Tableau 5

\begin{tabular}{|c|c|c|}
\hline$/ \mathbf{p} /$ & /' lapa / «lapa» & /b / / taba / «taba» \\
\hline $7 \mathbf{t}$ & T'lata / «lata» & / d / / seda / 《seda \\
\hline$/ \mathbf{k} /$ & /'takU / «taco» & /g / /'lagU / «lago» \\
\hline $7 \mathbf{f} /$ & / ka'fe / «café»» & /'lava / «lava» \\
\hline & T'lasU / «laço» & $/ \mathbf{z} /$ / kaza / «casa» \\
\hline $7 \int /$ & T'mefa / «mecha» & /'lazI / «lage» \\
\hline $\mathbf{m}$ & / kama / «cama» & / $\mathbf{\Lambda} /$ / kaKa / «calha» \\
\hline $7 \mathbf{n} /$ & T'tejU / «tenho» & /n / / pena / «pena» \\
\hline 711 & T'kola / «cola» & \\
\hline
\end{tabular}

En finale absolue seuls figurent les archiphonèmes / $\mathbf{R} /$, / $\mathbf{Z}$ / et / W / résultant de la neutralisation des oppositions entre les phonèmes $/ \mathbf{r}, / \mathbf{s} /, / \mathbf{z} /, / \mathbf{l} /$.

\section{Les voyelles et l'accent}


Toutes les voyelles, nasales et orales, sont admises en position tonique et prétonique; en position postonique seules figurent les voyelles / a /, / ع /, / i /, / u /. En position postonique les oppositions $/ \boldsymbol{\varepsilon} / \sim / \mathbf{e} /$ et $/ \supset / \sim / \mathbf{o} /$ se neutralisent : elle sont représentées par les archiphonèmes / $\mathbf{E} /$ et / $\mathbf{O} /$.

\section{Voyelles toniques}

Tableau 6

\begin{tabular}{|c|c|}
\hline / a / / 'mala / «mala» & / 'vela / \\
\hline / e / / 'pelU / «pelo» & / 'vila / \\
\hline / o / / 'bola / «bola» & / 'lobU / \\
\hline / u / / 'luva / «luva» & / 'mãta / \\
\hline / ̃̃ / / pẽtI / «pente» & / 'sĩkU / \\
\hline / $\tilde{\mathbf{o}} /$ / 'kõta / «conta» & / $\tilde{\mathbf{u}} / \quad$ / 'nũka / «nunca» \\
\hline
\end{tabular}

Voyelles pré-toniques

Tableau 7

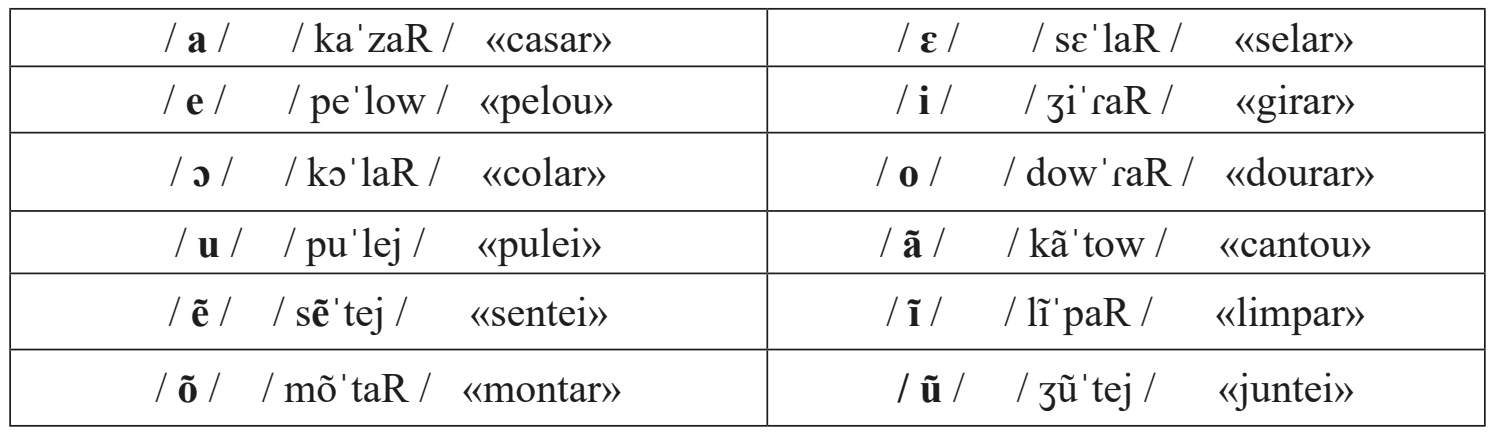

\section{Voyelles post-toniques}

Tableau 8

\begin{tabular}{|c|c|}
\hline / a / / 'lãpada / «lâmpada» & / $\boldsymbol{\varepsilon} /$ / 'batcga / «bátega» \\
\hline / u / / 'makula / «mácula» & / i / / sadika / «sádica» \\
\hline
\end{tabular}




\section{Réalisation et distribution des phonèmes}

Outre les phénomènes de neutralisation signalés, la réalisation des phonèmes du parler Paraíbano connaît un grand nombre des variantes, libres et combinatoires, très fréquentes et distribuées sur l'ensemble de l'espace enquêté.

Les variantes libres, malgré leur nom, reflètent toujours la norme régionale de Paraíba; les variantes combinatoires sont liées au contexte phonique qui les entoure.

Les phonèmes $/ \mathrm{a} /, / \mathrm{e} /, / \mathrm{i} /, / \mathrm{o} /, / \mathrm{u} /$ ont des variantes nasalitées devant les consonnes nasales qui les suivent : elles n'ont pas un statut de phonèmes.

\section{Phonèmemes, archiphonèmes et leurs réalisations}

Tableau 9

\begin{tabular}{|c|c|c|}
\hline PHONÈMES & ARCHIPHONÈMES & RÉALISATIONS \\
\hline $\begin{array}{c}\mathbf{e} / \\
/ \text { Edu'kaR/ }^{\prime} / \\
/ \boldsymbol{\varepsilon} /\end{array}$ & $/ \mathbf{E} /$ & $\begin{array}{c}\text { e] }] \text { edu kaf] } \\
\text { «educar» } \\
{[\varepsilon][\varepsilon d u ' k a h]}\end{array}$ \\
\hline $\begin{array}{l}/ \mathbf{e} / \\
/ \text { IS 'tow/ } \\
/ \mathbf{i} / \\
\end{array}$ & / I / & $\begin{array}{c}{[\mathbf{e}][\text { e ' tow] }} \\
\text { «estou» } \\
\text { [i] [i ['tow] }\end{array}$ \\
\hline $\begin{array}{c}\mathbf{0} / \\
/ \text { pOR 'tãw / }\end{array}$ & / O / & $\begin{array}{c}\text { [o] [poh'tãw] } \\
\text { «portão» } \\
\text { [ग] [pэhtão] }\end{array}$ \\
\hline $\begin{array}{c}\text { / o / } \\
\text { / bU'tãw / } \\
\text { / u / }\end{array}$ & / U / & $\begin{array}{c}\text { [o] [bo' tãw] } \\
\text { «botão» } \\
\text { [u] [bu'tãw] }\end{array}$ \\
\hline 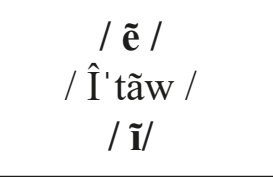 & / $\mathbf{\mathbf { I }} /$ & $\begin{array}{c}\text { [ẽe] [ẽ' tãw] } \\
\text { «então» } \\
\text { [i] [i' tãw] }\end{array}$ \\
\hline $\begin{array}{c}/ \mathbf{l} / \\
/ \text { 'saWtU / } \\
/ \mathbf{w} /\end{array}$ & / W / & $\begin{array}{c}\text { [1] ['sałtu] } \\
\text { «salto» } \\
\text { [w]['sawtu] }\end{array}$ \\
\hline $\begin{array}{c}\text { / s / } \\
\text { / beSta / } \\
/ \mathbf{J} /\end{array}$ & / S / & $\begin{array}{c}\text { [s]['besta] } \\
\text { «besta» } \\
\left.\text { [J] [' be } \int \mathrm{ta}\right]\end{array}$ \\
\hline $\begin{array}{c}/ \mathbf{s} / \\
/ \text { 'mewZ'peS / } \\
/ \mathbf{z} /\end{array}$ & / Z / & $\begin{array}{c}\text { [s] ['mews 'prjs] } \\
\text { «meus pés» } \\
\text { «meus olhos» } \\
\text { [z] [' mew' zoKus] }\end{array}$ \\
\hline
\end{tabular}




\begin{tabular}{|c|c|c|}
\hline $\begin{array}{c}\text { / } \tilde{\mathbf{o}} / \\
\text { / } \tilde{\mathrm{O}}^{\prime} \operatorname{padrI} / \\
/ \tilde{\mathbf{u}} /\end{array}$ & / $\tilde{\mathbf{O}}$ / & 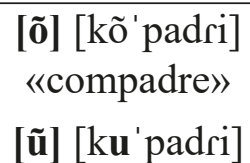 \\
\hline $\begin{array}{c}/ \mathrm{r} / \\
\text { / noRtI / } \\
/ \mathbf{r} /\end{array}$ & / $\mathbf{R}$ / & $\begin{array}{c}\text { / / / ['norti] } \\
\text { «norte» } \\
/ \mathbf{r} / \text { ['norti] }\end{array}$ \\
\hline
\end{tabular}

\section{Variantes régionales de Paraíba}

\section{a) Variantes libres}

1. [E] Réalisation de l'archiphonème / $\mathrm{E} /$ :

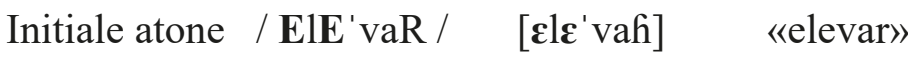

Interne atone / REgu'laR/ [regu'laf] «regular»

Interne tonique /'lEvI / ['levi] «leve»

2. [i] Réalisation de 1'archiphonème / I/, dans les positions suivantes :

Initiale atone / IS' tow / [if'tow $]$ «estou»

Interne atone / dI'zezU / [di'zezu ] «desejo»

Finale atone / 'nojtI / ['nojti] «noite»

3. [0] Réalisation de l'archiphonème / $\mathrm{O} /$, dans les positions suivantes :

Initiale atone / Obri'gadU / [obri'gadu] «obrigado»

Interne atone / $\quad$ kO'braR / [ko'braf] «cobrar»

4. [u] Réalisation de l'archiphonème / U /, dans les positions suivantes :

Initale atone / U'veKa / [u'veKa] «ovelha»

Interne atone /pU'risU / [pu'risu] «por isso»

Finale atone / 'bobU / ['bobu] «bobo»

5. [w] Réalisation de l'archiphonème / W /, dans les positions suivantes :

Interne tonique / RE'zoWvI / [he'zowvi] «resolve»

Interne atone / maW'vada / [maw'vada] «malvada»

Finale tonique / fu'ziW / [fu'ziw] «fuzil»

Finale atone / fasjW / ['fasjw] «fácil»

6. [J] Réalisation de l'archiphonème / S /, dans les positions suivantes :

Interne tonique / 'baSta / ['bafta $]$ «basta»

Interne atone /goS'tozu / [gof'tozu] «gostoso»

7. [h] Réalisation de l'archiphonème / R/, dans les positions suivantes : 


$\begin{array}{llll}\text { Initiale tonique } & / \text { 'RadjU / } & {[\text { 'hadju }]} & \text { «rádio» } \\ \text { Initiale atone } & / \mathrm{RE} \text { 'lozjU / } & {[\text { he'lozju }]} & \text { «relógio» } \\ \text { Interne tonique } & / \text { 'noRtI / } & {[\text { 'nofiti }]} & \text { «norte» } \\ \text { Interne atone } & / \text { nOR'maW / }[\text { 'maw }] & \text { «normal» } \\ \text { Finale tonique } & / \text { ko'meR / } & {[\text { kô'mef }]} & \text { «comer» }\end{array}$

8. [ĩ] Réalisation de l'archiphonème / Î /, normal dans le langage populaire, dans les positions suivantes :

Initiale atone

$$
\begin{array}{llll}
\text { Initiale atone } & / \hat{\mathbf{I}}^{\prime} \text { tãw } / & {\left[\tilde{1}^{\prime} \text { tãw }\right]-} & \text { 《então» } \\
\text { Interne atone } & / \text { dIzÎI'ganU / } & {[\text { diz Ĩ'gânu }]} & \text { «desengano» }
\end{array}
$$

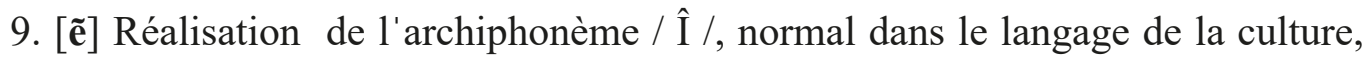
dans les positions suivantes :

$\begin{array}{llll}\text { Initiale atone } & / \hat{I}^{\prime} \text { tãw / } & {\left[\tilde{e}^{\prime} \text { tãw }\right]} & \text { «então» } \\ \text { Interne atone } & / \text { dez I' 'ganu / } & {[\text { dezẽ'gânu }]} & \text { «desengano» }\end{array}$

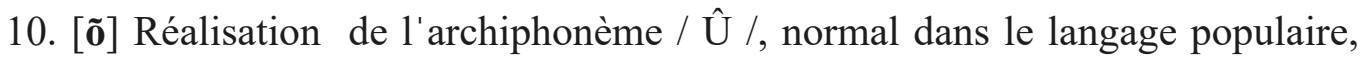
dans la position suivante :

Interne atone / k̂́'padrI / [kõ'padri] «compadre»

11. [ũ] Réalisation de l'archiphonème / $\hat{U}$ /, normal dans le langage populaire, dans la position :

Interne atone / kÛ'padrI/ [kũ'padri] «compadre»

\section{b) Variantes combinatoires}

1. [â] Réalisation nasalisée du phonème / a / devant consonnes nasales :

$\begin{array}{lcll}\text { Initiale tonique } & \text { /'ama / } & {[\text { 'âma }]} & \text { «ama» } \\ \text { Initiale atone } & / \text { a'maR / } & {[\text { â'maf }]} & \text { «amar» } \\ \text { Interne tonique } & / \text { 'bana / } & {[\text { 'bâna }]} & \text { «banha» } \\ \text { Interne atone } & / \text { ka'ncla / } & {[\text { kâ'ncla }]} & \text { «canela» }\end{array}$

2. [êe] Réalisation nasalisée du phonème / e / devant consonnes nasales :

$\begin{array}{llll}\text { Initiale atone } & \text { / emi'graR / } & \text { [êmi'graf] } & \text { «emigrar» } \\ \text { Initiale tonique } & / \text { 'ema / } & \text { ['êma] } & \text { «ema» } \\ \text { Intrne atone } & / \text { te'meR / } & \text { [tê'mef] } & \text { «temer» } \\ \text { Interne tonique } & / \text { 'lema / } & \text { ['lêma } & \text { «lema» }\end{array}$

3. [î] Réalisation nasalisée du phonème / i / devant consonnes nasales : 


$\begin{array}{llll}\text { Initiale atone } & \text { / i'nûmEruS / } & \text { [î'nûmerus] } & \text { «inúmeros» } \\ \text { Initiale tonique } & \text { / 'imã / } & \text { ['îmã] } & \text { «imã» } \\ \text { Interne atone } & \text { / si'mẽtU / } & \text { [sî'mẽtu] } & \text { «cimento» } \\ \text { Interne tonique } & \text { / mi'ninU / } & \text { [mî'nînu] } & \text { «menino» }\end{array}$

4. [0] Réalisation nasalisée du phonème / o / devant consonnes nasales:

$\begin{array}{llll}\text { Initiale tonique } & \text { / 'omẽj / } & \text { ['ômẽj] } & \text { «homem» } \\ \text { Initiale atone } & \text { / o'neStU / } & \text { [ô'neftu] } & \text { «honesto» } \\ \text { Interne tonique } & \text { / a'roma / } & \text { [a' rôma] } & \text { «aroma» } \\ \text { Interne atone } & \text { / akomod'aR / [akômod'ah] } & \text { «acomodar» }\end{array}$

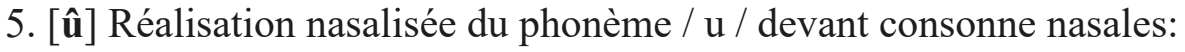

$\begin{array}{llll}\text { Initiale tonique } & \text { / 'unika / } & \text { ['ûnika] } & \text { «única» } \\ \text { Initiale atone } & \text { / uni'ãw / } & \text { [ûni'ãw] } & \text { «união» } \\ \text { Interne tonique } & \text { / a'lunU / } & \text { [a'lûnu] } & \text { «aluno» } \\ \text { Interne atone } & \text { / asu'miR / } & \text { [asû'mif] } & \text { «assumir» }\end{array}$

6. [s] Réalisation de l'archiphonème / S /, devant consonne sourde ou en finale :

$\begin{array}{llll}\text { Interne tonique } & \text { / 'kaSpa / } & \text { ['kaspa] } & \text { «caspa» } \\ \text { Interne atone / } & \text { Ju'viSkU / } & \text { [ Ju'visku] } & \text { «chuvisco» } \\ \text { tonique } & \text { / Ra'pajS / } & \text { [ha' } \text { ' pajz] } & \text { «rapaz» } \\ \text { atone } & / \text { 'pasuS / } & \text { ['pasus }] & \text { «passos» }\end{array}$

7. [z] Réalisation de l'archiphonème / Z /, devant consonne sonore :

Interne /'neZga / ['nezga] «nesga»

\section{Diphtongaisons}

Les phonèmes vocaliques suivis de / $\mathbf{s} /$ ou / $\mathbf{z} /$, dans les mots monosyllabiques et

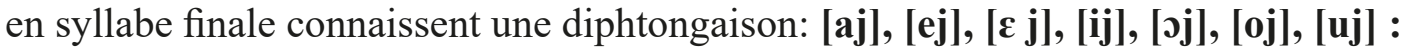

Tableau 10

\begin{tabular}{|c|c|c|}
\hline 1.as & $\begin{array}{c}/ \mathrm{aS} />\text { [ajs }] \quad-\langle\text { mas } » \\
/ \mathrm{maS} />\text { [majs }]\end{array}$ & $\begin{array}{c}\text { 7.iz } / \text { iS / > [ijz] - «fiz» } \\
\text { / fiS / > [fijz }]\end{array}$ \\
\hline 2.az & $\begin{array}{c}\text { / aS / > [ajs] } \quad-\text { «rapaz» } \\
\text { / Ra'paS / > [ha'pajs] }\end{array}$ & $\begin{array}{c}\text { 8. os } \quad /(\mathrm{S} />\text { [jjs }]-\text { «vós» } \\
/ \text { voS / > [vojs }]\end{array}$ \\
\hline & $\begin{array}{cl}\text { 3.es } & / \text { eS } />\text { ejs }]- \text { «três» } \\
& / \text { tres } />\text { trejs }]\end{array}$ & $\begin{array}{cl}9.0 & / \mathrm{oS} />\text { [ojs] - «pôs» } \\
& / \mathrm{poS} />\text { [pojs] }\end{array}$ \\
\hline & $\begin{array}{c}/ \text { eS } />[\text { ejz }] \\
\qquad / \mathrm{feS} />[\text { fejs }]\end{array}$ & $\begin{array}{c}\text { 10. oz / oS / > [ojz }]- \text { «arroz» } \\
\text { / a' hoS / > [a' hojz }]\end{array}$ \\
\hline
\end{tabular}




\begin{tabular}{|c|c|}
\hline 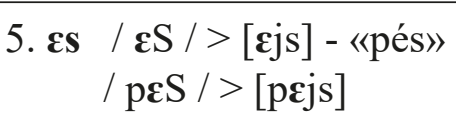 & $\begin{aligned} \text { 11. us } / \mathrm{uS} />[\text { ujs }]-\langle\text { pus» } \\
/ \mathrm{puS} />[\text { pujs }]\end{aligned}$ \\
\hline $\begin{array}{c}\text { 6. is } \quad / \mathrm{iS} />[\mathrm{ijs}]-\text { - «quis» } \\
/ \mathrm{kiS} />[\mathrm{kijz}]\end{array}$ & $\begin{array}{l}\text { 12. } \mathbf{u z} / \mathrm{uS} />\text { [ujz] - }\langle\text { reduz» } \\
/ \mathrm{R} \boldsymbol{\varepsilon}^{\prime} \mathrm{duS} />\left[\text { h } \boldsymbol{\varepsilon}^{\prime} \text { dujz }\right]\end{array}$ \\
\hline
\end{tabular}

\section{Monophtongaison}

Dans certains contextes, il apparaît le phénomène de la monophtongaison, ce qui réduit considérablement le nombre des diphtongues, dans la façon de parler Paraíbana.

/ aj / > [a]

En position interne, devant $/ \int /$, / aj / se réalise [a]

«caixa» /'kajfa/ ['kaja]

/ ej / > [e]

En position interne, devant / $\mathrm{r} /, \mathrm{J} / \mathrm{e} / \mathrm{z} / \mathrm{,} / \mathrm{ej} / \mathrm{se}$ réalise comme $[\mathrm{e}]$

«beirada» / bej'rada / [be'rada]

«deixar» / / dej'faR/ [de'fah]

«beijar» / bej'zaR/ [be'zah]

/ ej / > [e] ou [ej]

En position interne, devant / t / ou / g /, / ej /-peut se réaliser comme [e] ou se maintenir comme [ej]

$\begin{array}{lll}\text { «colheita» } & \text { / kUK' 'ejta / } & {[\mathrm{ku} \text { 'Keta }]} \\ \text { «leitão» } & / \text { lej'tãw / } & {[\text { lej'tãw }]} \\ \text { «manteiga» } & \text { / mã'tejga / } & \text { [mã'tega }]\end{array}$

/ ow / > [o] ou [u]

En position initiale tonique / ow / se réalise comme [o] ; à l'initiale atone comme [u]:

$\begin{array}{lll}\text { «ouro» } & \text { / 'owrU / } & \text { ['oru }] \\ \text { «ouvido» } & \text { / ow'vidU / } & {[\text { ' ' 'vidu }]}\end{array}$

/ ow / > [o]

En position inerne / ow / se réalise comme [o]

«besouro» / bI'zowrU / [bi'zoru]

/ ul / uW / > [u]

En position initiale, interne et finale, / uW / se réalise comme [u]

«último» / 'uWtimU / ['utimu]

«pulmão» / puW'mãw / [pu'mãw]

«sul» /'suW / ['su]

Vol. 25 - Ano $44-n^{\circ} 1-2020$ 


$$
\text { / jo / > [u] }
$$

En position finale / jo / se réalise comme $[\mathrm{u}]$

«negócio» / n $\varepsilon^{\prime}$ gosjU / [nع'gosu]

/ ja / > [a]

En position finale / ja / se réalise comme [a]

«farmácia» / faR'masja / [fah'masa]

/ je / > [i]

En position finale / je / se réalise comme [i]

«planície» / pla'nisje / [plâ'nisi]

D'autres phénomènes phonétiques fréquents dans le parler Paraíbano ont été observés, parmi lesquels : la chute des phonèmes / / /, / / /, / $\mathbf{s} /$ en position finale absolue ; la réduction du groupe / nd / > / n /; le iotacisme de / j /, / K/, / r/; la laryngalisation du phonème $/ \mathrm{s} />/ \mathrm{h} /$ en finale de syllabe interne.

\section{Conclusion}

Publiés il y a plus de trente ans (1984), les deux premiers volumes de l'Atlas de Paraíba occupent, chronologiquement, la troisième place dans la série des Atlas 'régionaux' du Brésil. Ils ont sans doute contribué à la connaissance du phonétisme et du lexique, en particulier, des parlers de cet Etat. Il a permis de tester une nouvelle représentation cartographique des données lexicales qui ne sera pas adoptée, cependant, par les projets atlantographiques qui suivront, à cause des problèmes qui ont été évoqués. Il a permis aussi de mettre en évidence des traits phonétique, morphosyntaxiues et lexicaux qui pouvaient être considérés comme caractéristiques de l'espace enquêté ne connaissant pas, à l'époque, la situation des autres régions, à commencer par les régions environnantes. La réalisation d'autres Atlas et, surtout celle de l'ALiB, pourra apporter une réponse définitive à cette question.

\section{Références}

AGUILERA VANDERCI de A. Atlas linguístico do Paraná, Londrina, Universidade Estadual de Londrina, 1993.

ARAGÃO Maria do Socorro Silva de et BEZERRA de MENEZES Cleusa P., Atlas linguístico da Paraíba, Brasília, UFPB/CNPq, Coordenação Editorial, 1984, v. 1, 2.

ARAGÃO Maria do Socorro Silva de, «Aspectos léxicos do atlas linguístico da Paraíba», Estudos Linguísticos e Literários, 5, Salvador, UFBA, 1986, pp. 129-150. 
ARAGÃO Maria do Socorro Silva de, «Aspectos fonético-fonológicos do atlas linguístico da Paraíba», XVIII C Congrès International de Linguistique et de Philologie Romanes, Université de Trèves (Trier), 1986, Tübingen. Max Niemeyer Verlag, 1991, pp. 549-560.

ARAGÃO Maria do Socorro Silva de, «A despalatalização e iotização no atlas linguístico da Paraíba», II Encontro Nacional de Fonética e Fonologia, Anais, Niterói, UFF, 1992.

ARAGÃO Maria do Socorro Silva de, «Aspectos fonéticos do atlas linguístico da Paraíba - As vogais pretônicas», IX Encontro Nacional da Anpoll, Anais, João Pessoa. ANPOLL, 1995, v.2, Tomo I, pp. 833-840.

BRANDÃO Sílvia de F., A geografia linguística no Brasil, São Paulo, Ática, 1991.

FERREIRA Carlota et al. Atlas linguístico de Sergipe, Salvador, UFBA - Instituto de Letras/Fundação Estadual de Cultura de Sergipe, 1987.

FERREIRA Carlota et al., Diversidade do português do Brasil : estudos de dialetologia rural e outros. Salvador, UFBA, 1994.

NASCENTES Antenor, Bases para a elaboração de atlas linguístico do Brasil, Rio de Janeiro, Casa de Rui Barbosa, 1958.

NASCENTES Antenor, O idioma nacional, Rio de Janeiro, Livraria Acadêmica, 1960.

ROSSI Nelson. Atlas prévio dos falares baianos, Rio de Janeiro, INL, 1963.

SILVA NETO Serafim, Guia para estudos dialetológicos, Florianópolis, [s. ed.], 1955.

ZÁGARI Mário Roberto L. et al., Esboço de um atlas linguístico de Minas Gerais, Rio de Janeiro, Fundação Casa de Rui Barbosa, 1977. 